\title{
An Optimal Generalized Autoregressive Conditional Heteroscedasticity Model for Forecasting the South African Inflation Volatility
}

\author{
Ntebogang Dinah Moroke, Albert Luthuli \\ North West University, South Africa \\ University Drive, RSA \\ Ntebo.Moroke@nwu.ac.za
}

\begin{abstract}
In most cases, financial variables are explained by leptokurtic distribution and often fail the assumption of normal distribution. This paper sought to explore the robustness of GARCH-type models in forecasting inflation volatility using quarterly time series data spanning 2002 to 2014. The data was sourced from the South African Reserve Bank database. SAS version 9.3 was used to generate the results. The initial analyses of data confirmed non-linearity, hereroscedasticity and non-stationarity in the series. Differencing was imposed in a log transformed series to induce stationarity. Further findings confirmed that $A R(1)$ IGARCH $(1,1)$ model suggested a high degree persistent in the conditional volatility of the series. However, the $A R(1) \_E G A R C H(2,1)$ model was found to be more robust in forecasting volatility effects than the $A R(1) \_I G A R C H(1,1)$ and $A R(1) \_G J R-G A R C H(2,1)$ models. This model confirmed that inflation rates in South Africa exhibits the stylised characteristics such as volatility clustering, leptokurtosis and asymmetry effects. These findings may be very useful to the industry and scholars who wish to apply models that capture heteroscedastic and non-linear errors. The findings may also benefit policy makers and may be referred to when embarking on strategies in-line with inflation rate.
\end{abstract}

Keywords: GARCH_type models, inflation rates, volatility, heteroscedasticity

\section{Introduction}

The most commonly used statistical model is regression analysis. The standard assumptions associated with this model are, however, violated when applied to time series data. The problem of autocorrelation is common in many time series data and this could be taken care of only when the correct order of lags is chosen. Literature suggests several methods to take care of autocorrelation errors in a model. This study uses the univariate models which have the capacity to take care of these problems. The proposed methods are further effective when applied to data which exhibit heteroscedastic and non-linear errors. These assumptions may in many practical applications not be realistic. Time series data is collected over certain intervals, short time or long time intervals causing the associated errors to be time dependent and correlated. It is thus important to use a model that incorporate the possibility of non-constant error variance known a heteroscedasticity model. In the main, this study presents generalized autoregressive conditional heteroscedasticity (GARCH) models introduced by Bollerslev (1986) and explores their predictive power in a series containing heteroscedastic errors. The methods are applied in inflation data of South Africa. Inflation measures the persistent and continuous rise in the general price levels in an economy. Webster (2000) describes inflation as the persistent increase in the level of consumer prices or persistent decline in the purchasing power of money. This is one economic factor that affects all other levels of the economy, and as a result it is the duty of every country to have effective control this sector. Suleman and Sarpong (2012) highlighted that most departments use inflation rates as basis of argument when debating on the state of the economy. Still on the same breadth, David (2001) described inflation as one of the main focus of economic policy in the entire world. Accurate future forecasts of inflation must be available so that they are incorporated in decisions affecting other sectors of the economy especially those associated with business transactions. The findings of this study would further aid in efficient allocation of economic resources in the country. This would also boost the rate of economic growth by encouraging savings to finance investments by both the government and households. 
The empirical methods proposed uses the univariate time series analysis which captures both the present and past values of a unit series. The methods also have the ability to capture the volatility effects and can accommodate the dynamics of conditional heteroscedasticity and non-linearity. It is important to handle heteroscedasticity with appropriate non-constant variance models since it has effects on the accuracy of forecasts of confidence limits (Amos, 2010). Various models from the GARCH family would be explored and the one that passes the diagnostic tests will be considered for further analyses. The empirical findings would be applicable to practitioners in the industry, policy makers in the government, businesses and the entire community of South Africa. Better planning would be done once the forecasts are known. Scholars would also benefit from this study as the results would contribute by either closing or bridging a gap in existing literature. The results would also serve as a basis for further studies to all interested parties.

There is a dearth of literature which reports on studies that explored the ability of GARCH type models to estimate inflation volatility in the context of South Africa. Most of the studies about inflation applied the BoxJenkins autoregressive moving average (ARMA) models. There is a gap in literature in the context of South Africa on studies that modelled and forecasted inflation volatility with GARCH-type models. To the best of my knowledge, the only study that modelled and forested inflation volatility is one conducted by Amos (2010). This study applied SARIMA $(1,1,0)(0,1,1)$ and $\operatorname{GARCH}(1,1)$ models on inflation rates of South Africa. Even though the two models were found to be good predictors of inflation rate, the GARCH $(1,1)$ yielded least forecast errors and proved to be superior to the SARIMA model. This is a confirmation that the GARCH $(1,1)$ model is robust in producing forecasts of inflation rates and capturing variations in the data; the task which a SARIMA model failed to fulfil. Traditional time series models such as SARIMA assumes constant variances in the series. Conversely, most of the economic and financial series to a large extent exhibit non constant conditional variance (are heteroscedastic) and the analysis of such data is better handled with the novel GARCH-type models. Hence, the current study proposes and explores the GARCH-type framework to model and forecast inflation volatility.

A similar study to Amos (2010) was undertaken in Nigeria by Osarumwense and Waziri (2013). This study explored the theory of univariate non-linear time series and applied both ARIMA and GARCH models to nonstationary series. One of the conditions under which ARIMA can perform very well besides the linearity and constant variance is when the data used does not have unit root. The authors used these models under wrong conditions since they disregarded the most important assumptions. However, both models passed the battery of diagnostic tests and GARCH $(1,0) \_$ARIMA $(1,0)$ were found to be sufficient to estimate Nigeria's inflation volatility for the periodJanuary 2012 to December 2013. Other empirical studies that considered the use of GARCH models in the area of inflation were carried out in Ghana such as those by Alnaa and Ahiakpor (2011) and Suleman and Sarpong (2012). These studies used both seasonal and non-seasonal models that assume constant variance just like Amos (2010) and disregarded the issue of conditional heteroscedasticity of the series in inflation data. To prove that the GARCH models are suitable for modelling the series with heteroscedastic errors, the comparative study by Awogbemi and Oluwaseyi (2011) favoured the GARCH model over the conventional ARIMA model. Similarly, Nortey et al. (2014) compared the performance of autoregressive conditional heteroscedasticity (ARCH), GARCH and exponential GARCH (EGARCH) models proposed by Nelson (1991) in modelling monthly rates of inflation in Ghana. The findings of this study showed that the EGARCH $(1,2)$ model with a mean equation of ARIMA $(3,1,2)+(0,0,0) 12$ was appropriate formodelling Ghana's monthly rates of inflation.

One study that explored the varying volatility dynamics of inflation series was conducted by Perniaag et al. (2004) in Malaysia. Though this study did not consider variety of GARCH-types, a comparison was made between the GARCH and the exponential GARCH (EGARCH) models. The latter is not only suitable for capturing heteroscedastic errors; it is further used to capture the stochastic variations and asymmetries in the data. Though the diagnostic tests of these models provided significant results, EGARCH was chosen as the best model due to the nature of inflation data which is explained by highly irregular fluctuations. This shows that GARCH-type models have the ability to capture and reveal varying characteristics associated with time series data. Su (2010) studied financial volatility in Ghana using the GARCH and EGARCH models. Empirically, the results suggested EGARCH model as a good model of sample data than the ordinary GARCH model. EGARCH in this study modelled the volatility of Chinese stock returns better than the GARCH. The GARCHtype models are also useful time varying models in capturing the heteroscedastic errors in higher order levels 
such as the bivariate and multivariate time series. Several studies applied these models to study the effect of other financial and/or economic variables on inflation rates of different countries. Such studies include those by Igogo (2010) who in the first place used the GARCH $(1,1)$ model and realised such model violates the nonnegativity condition. To remedy the problem, $\operatorname{EGARCH}(1,1)$ model was employed and was found to be adequate measure of volatility in the series.

Likewise, Chong et al. (2002) applied the GARCH and the GARCH-in-the-mean type models in their study on exchange rate volatility. Just like the inflation rate, exchange rate share similar properties of conditional variance and non stationarity and as such same kind of time series models are applicable to such data sets. The findings of this study did not favour the null hypothesis of constant variances in the exchange rate implying the applicability of the GARCH-type models on the series. Consequently, the GARCH-in-the-mean was favoured over the GARCH model as it modelled heteroscedasticity of daily exchange rates better. Mokoma and Moroke (2015) also applied the multivariate ARCH approach to study exchange rate volatility. Three other financial variables such as the interest rates, inflation rates and GDP were used as determinants of exchange rate volatility in their study. The $A R C H$ (1) was chosen over the $A R C H$ (2) model since the former proved to reveal volatility of the series much better than the latter. Another study that applied GARCH models to inflation and inflation uncertainty relationship include one by Jehovanes (2007). It is clear according to evidence recorded from the literature reviewed that the GARCH models are suitable to model and forecast time series data with conditional variance. EGARCH model appears to be the most favourable model to use in modelling data with heteroscedastic errors. The exploration of these models will allow the researcher to suggest an optimal GARCH-type model from the list and use it to produce future forecasts of inflation rates in the context of South Africa.

\section{Methodology}

Data: The data used in this study comprises50 quarterly observations of the South African inflation expectation of the current year. These data covers the period from the third quarter of 2002 to the fourth quarter of 2014. The Consumer Price Index (CPI) is published by the Statistics South Africa and the South African Reserve Bank. Due to the nature of these data, the stochastic properties are expected to be nonstationary. The stationarity of the series is confirmed by assessing the first difference of the log transformed series. The desire is to have the stochastic properties of the series oscillating around the zero line. If this is not a case, the series is non-stationary. To convert the prices to returns, logarithmic transformations will be used. The logarithmic return is based upon the following mathematical definition;

$$
r_{t}=\ln \frac{P_{t}}{P_{t-1}},
$$

Where $r_{t}$ the return is for any time, $t, P_{t}$ is the CPI value at time, $t$ and $P_{t-1}$ is the CPI value at time, $t-1$.The Statistical Analysis Software (SAS) version 9.3, registered to the SAS Institute Inc. Cary, NC, USA will be used for data analysis

Model identification and selection: Most econometric models, especially those utilising the ordinary least square method, are built on the assumption of constant variance. However, supporters of the efficient market hypothesis claim that financial data are random and as such makes any speculation based on past information fruitless (Frimpong and Oteng-Abayie, 2006). In essence, the basic model for estimating volatility using the ordinary least squares method is the naiverandom walk model which is analogous to the standard regression model assuming uncorrelated error given as;

$$
Y_{t}=\mu+X_{t}^{\prime} \beta+\varepsilon_{t},
$$

Where $\mu$ is a mean value which is expected to be insignificantly different from zero, $\varepsilon_{t}=n_{t}$ and $n_{t}$ are uncorrelated but have non-constant variance. Following Engle (1982), assume that the error term can be modeled as;

$$
n_{t}=\sigma_{t} e_{t},
$$


Where $e_{t}$ are identically and independently distributed(i.i.d.) random variables with mean 0 and variance 1 , independent of past realizations of $n_{t-i}$, and;

$$
\sigma_{i}^{2}=\theta_{0}+\theta_{1} n_{t-1}^{2}+\cdots+\theta_{s} n_{t-s}^{2}
$$

Given all the information up to time $(t-1)$, the conditional variance of the $n_{t}$ becomes;

$$
\operatorname{Var}_{t-1}\left(n_{t}\right)=E_{t-1}\left(n_{t}^{2}\right)=E\left(n_{t}^{2} \mid n_{t-1, \ldots}\right)=\sigma_{t}^{2},
$$

and is related to the squares of past errors, and changes over time. Large errors through $n_{t-i}^{2}$ gives rise to variance, which tends to be followed by another large error. This is a phenomenon of volatility clustering and is common in many financial time series (Wei, 2006).Equation [4] is simply the optimal forecast of $n_{t}^{2}$ if $n_{t}^{2}$ follows the following AR (s) model;

$$
n_{t}^{2}=\theta_{0}+\theta_{1} n_{t-1}^{2}+\cdots+\theta_{s} n_{t-s}^{2}+a_{t},
$$

Where $a_{t}$ is an $N\left(0, \sigma_{a}^{2}\right)$ white noise process. Engle (1982) as a result called the model of error term $n_{t}$ with the variance specification given in [2] and [3] the ARCH model or ARCH(s) model. Equation [3] containing the property [4] is given as;

$$
\sigma_{t}^{2}=\theta_{0}+\phi_{1} \sigma_{t-1}^{2}+\cdots+\phi_{r} \sigma_{t-r}^{2}+\theta_{1} n_{t-1}^{2}+\theta_{s} n_{t-s}^{2}
$$

is known as the GARCH model of order $(r, s)$. Equation [7] is an ARMA form for the squared series $a_{t}^{2}$. As a result, a GARCH model can be regarded as an application of the ARMA idea to the squared series $a_{t}^{2}$ (Tsay, 2005). Other GARCH-family models can be constructed from [7]. For instance, if the AR polynomial of the GARCH representation [7] has a unit root, then we have an integrated GARCH (IGARCH) model, i.e. $\left(1-\alpha_{1}-\cdots-\alpha_{m}\right)=0$. From [4] and [7], it is evident that in specification of the ARCH, GARCH and IGARCH models, the effect of errors on conditional variance is symmetric, implying that positive error has the same effect as the negative error of the same magnitude (Wei, 2006). Moreover, because the $\sigma_{t}^{2}$ and $n_{t}^{2}$ are always non-negative, some restrictions on the coefficients $\theta_{0}$, and $\phi_{1}$ have to be imposed. In order to accommodate the symmetric relationship between financial variables, and their volatility changes and further to relax the restriction on the coefficients in the model, Nelson (1991) proposed that;

$$
\log \left(\sigma_{t}^{2}\right)=\gamma_{t}+\sum_{j-0}^{\infty} \psi_{j} g\left(a_{t-1-j}\right)
$$

Where $\psi_{0}=1$ and $a_{t}=n_{t} / \sigma_{t}$. Clarified by Nelson (1991), the function $g$ is chosen to allow for symmetric changes depending on the sign of $a_{t}$ in the conditional variance, $i . e$., we may choose

$$
g\left(a_{t}\right)=\delta a_{t}+\alpha\left(\left|a_{t}\right|-E\left|a_{t}\right|\right),
$$

When $0<a_{t}<\infty, g\left(a_{t}\right)$ is linear in $a_{t}$ with slope $\delta+\alpha$; when $-\infty<a_{t}<0, g\left(a_{t}\right)$ is linear with slope $\delta-\alpha$. consequently, [8] allows the conditional variance to respond asymmetrically to the rises and falls of the process. Equation [8] implies that the sign of $\sigma_{t}^{2}$ will be positive regardless of the sign of the coefficients (Nelson, 1991). Therefore the model in which the evolution of the conditional variance satisfies [8] is regarded by Nelson as the EGARCH.

Other asymmetric GARCH models are Glosten et al. (1993) and Zakoian (1994), GARCH (GJR-GARCH) also well known as Threshold GARCH, (Quadratic GARCH (QGARCH) (Engle and Ng, 1993) and Power GARCH (PGARCH) (Ding et al.1993). In the QGARCH model, the lagged errors' centres are shifted from zero to some constant values. There is an extra slope coefficient for each lagged squared error in the GJR-GARCH model and the PGARCH model does not only consider the asymmetric effect; it also affords alternative approach to model the long memory property in the volatility. The identification of the GARCH model could be done by examining the autocorrelation function (ACF) or the partial autocorrelation function (PACF). These functions 
also serve as a basis of the order specification of the GARCH models. The pattern of the ACFs and PACFs is compared with the theoretical characteristics of the ACF and PACF used to identify the orders of ARMA $(p, q)$ process.

Prior to fitting the GARCH model, it is also important to detect the presence of ARCH disturbances in time series data as suggested by Weiss (1984). This is one of the most important requirements for using the GARCH model. The author warned against overlooking the presence of heteroscedasticity as it does not only cause the ordinary least squares (OLS) estimates to be inefficient, but could also give rise to overparameterised ARMA model. This paper uses the Lagrange multiplier ( $L M)$ test proposed by Engle (1982) to ascertain the presence of $\mathrm{ARCH}$ disturbances. To be specific, the study tests the hypothesis that the conditional variances of the series are constant. The $L M$ test statistics are computed for various values of the $q$ lags. This test follows a chi-square distribution. If the $L M$ statistic is proven to be significant for higher lag orders, this is an indication that a GARCH model could parsimoniously predict the conditional error variance than a higher order ARCH. GARCH models have also been found to be effective when used on a series that exhibit non-linear effects (McLeod and Li, 1983). The presence of these effects may be checked with the Portmanteau Q-statistic. This statistic is based on a sample (ACF) of the squared OLS residuals for various lags and also follows a chi-square distribution. The desire is to obtain the significant observed probabilities associated with both the LM and the Q-statistics to be able to use the GARCH models. The selection of the most appropriate model for this study is done using Akaike Information Criterion $(A I C)$ and Bayesian Information Criterion(BIC)proposed by Faraway and Chatfield (1998).These measures are intended to identify model which best fits the data. The proposed formulae are given as;

$$
A I C=\frac{-2}{T} \ln (\text { likelihood })+\frac{2}{T} \times(\text { number of parameters }),
$$

Where the likelihood function is evaluated at a minimum likelihood estimates and $T$ is defined as a sample size (Tsay, 2010). To cater for the parameter estimation method used by the GARCH model, AIC reduces to;

$$
A I C=\ln \left(\bar{\sigma}_{\ell}^{2}\right)+\frac{2 \ell}{T}
$$

Where $\bar{\sigma}_{\ell}^{2}$ denotes the maximum likelihood estimate of $\sigma_{a}^{2}$ also known as the variance of $a_{t}$. Tsay (2005) explains the first term of this criterion as a measure of the goodness of fit of the model to the data. The second term is defined as the penalty function of the criterion. The author explained that this name is given to this criterion as it penalises the candidate model by the number of parameters used.

The proposed formula to represent the BIC is;

$$
B I C=\ln \left(\bar{\sigma}_{\ell}^{2}\right)+\frac{\ell \ln (T)}{T} .
$$

The penalty for each parameter used is 2 for AIC and $\ln (T)$ for BIC. On this basis, BIC has a tendency to select the model with the least lag more specifically when the sample size is moderate or large. In this study, the both the AIC and BIC are computed for $\ell=0, \ldots, P$, where $P$ is a prespecified positive integer. The optimal model order is chosen by the number of model parameters, which minimizes either AIC or BIC.

GARCH Model estimation: Parameters of the GARCH models can better be estimated with the method of maximum likelihood. During the estimation process, initial values of both the squared returns and past conditional variances are needed. Bollerslev (1986) and Tsay (2002) suggested the use of conditional variances in [7] or consequently, the past sample variance of the returns for the past variances may be used as initial values. Equation [2] is also taken into consideration when estimating the parameters of the model. From this equation, the $e_{t}$ are i.i.d.N $(0,1)$ and independent of past realisation of $n_{t-1}$. Equation [2] can alternatively be written in the following form to estimate the parameters;

$$
Y_{t}=X_{t}^{\prime} \beta+\frac{1}{1-\varphi_{1} B-\cdots-\varphi_{p} B^{p}} n_{t}
$$

or 


$$
n_{t}=\left(1-\varphi_{1} B-\cdots-\varphi_{p} B^{p}\right)\left(Y_{t}-X_{t}^{\prime} \beta\right) .
$$

Assuming $Y=\left(Y_{1}, \ldots, Y_{n}\right), X=\left(X_{1}, \ldots, X_{n}\right)$ and $Y_{0}$ and $X_{0}$ be some proper staring value required for computing $n_{t}$ for $t=1, \ldots n$ from [14]. The conditional maximum likelihood estimates can be obtained by maximizing the conditional log-likelihood given by;

$$
l=\log f\left(\beta, \varphi, \phi, \theta \mid Y, X, Y_{0}, X_{0}\right)=\prod_{t=1}^{n}\left(\frac{1}{2 \pi \sigma_{t}^{2}}\right)^{1 / 2} \exp \left(-\frac{n_{t}^{2}}{2 \sigma_{t}^{2}}\right),
$$

Where $\varphi=\left(\varphi_{1}, \ldots, \varphi_{p}\right), \phi=\left(\phi_{1}, \ldots, \phi_{r}\right)$ and $\theta=\left(\theta_{1}, \ldots, \theta_{s}\right)$.

Model diagnostics: Independence and normality of the residuals

The most recommended test for general departures from i.i.d. Observations are the BDS test introduced by Broock, Dechert and Scheinkman (1987). Since a single time series data is used in this study, only the univariate version of the BDS test is considered. If a given time series is independent, then no deterministic model is necessary for this completely random process; otherwise, there must exist some relationship in the series to be addressed. The BDS test of independence is based on the correlation dimension. Broock et al. (1997) follow-up study in 1996 show that the first-order asymptotic distribution of the test statistic is independent of the estimation error provided that the parameters of the model under test can be estimated $\sqrt{n}$ consistently.As a result, the BDS test can be used as a model selection tool and further as a specification test. The statistic has a standard normal distribution if the sample size is large enough. For small sample size, the distribution can be approximately obtained through simulation. The residuals of the model are rendered independent if the observed probabilities at for all embedding dimensions are in excess of 0.05 level of significance. The BDS test has stimulated quite a bulky literature and several applications have appeared in the finance area. See, Scheinkman and LeBaron (1989), Hsieh (1991) and Broock et al. (1991) for some of the application of the BDS test. To assess the normality of the residuals, the study uses a Kolmogorov-Smirnoff (KS) test. The desire is to obtain a symmetric approximated distribution of the residuals to conclude that the model fits the data. The KS test statistic must not be significant in order to render the model residuals normal.

Forecasting: The study further uses the model to find a forecast error variance that is heteroscedastic. The conditional variance of the GARCH model is obtained by taking the conditional expectation of the squared mean corrected returns (Ngailo, 2011). Firstly, note the optimal $l$-step ahead forecast of $Y_{t+1}$ given all information up to time $t$ is conditional expectation $E_{t}\left(Y_{t+l}\right)$. Wei (2006) suggested the associated $l-s t e p$ ahead forecast error as;

$$
\left(1-\varphi_{1} B-\cdots-\varphi_{p} B^{p}\right) \varepsilon_{t}=n_{t}
$$

Consequently,

$$
\begin{aligned}
& \varepsilon_{t}=\sum_{j=0}^{\infty} \psi_{j} B^{j} n_{t}, \text { and } \\
& \sum_{j=0}^{\infty} \psi_{j} B^{j}=\frac{1}{\left(1-\varphi_{1} B-\cdots-\varphi_{p} B^{p}\right)} .
\end{aligned}
$$

The $l-$ step ahead forecast error conditional variance thus becomes;

$$
\operatorname{Var}\left(\varepsilon_{t+1}\right)=\left(E_{t}\left[\varepsilon_{t+l}-E_{t}\left(\varepsilon_{t+l}\right)\right]^{2}=E_{t}\left[\sum_{j=0}^{l-l} \psi_{j} n_{t+l-j}\right]^{2},\right.
$$

The result of this equation is;

$$
E_{t}\left[\sum_{j=0}^{l-l} \psi_{j} \sigma_{i+l-j} e_{l+i-j}\right]^{2}=\sum_{j=0}^{l-l} \psi_{l}^{2} \sigma_{l+i-j}^{2},
$$


With $\sigma_{l+i-j}^{2}=E_{t}\left(n_{t+l-j}^{2}\right.$. As a result of these notations, Bollerslev (1986) suggested the following expression as a one-step ahead conditional forecast error heteroscedastic variance;

$$
\hat{\sigma}^{2}=\frac{1}{n} \sum_{i=1}^{n}\left(Y_{t}-X_{t}^{\prime} \widehat{\beta}^{2} .\right.
$$

Due to the difficulty in generating out-of-sample forecasts for GARCH models, or generating forecasts using non-linear models, only the in-sample-forecasts performances of the models will be examined. The measures of error forecast such as the mean absolute error (MAE), mean absolute percentage error (MAPE) and the mean square error (MSE) are used to evaluate the models for forecast ability. The model which generates least forecast error would be preferred most.

\section{Data Analysis}

Preliminary Analysis: This section presents the results of the 50quarterlyobservations on inflation rates from 2002 to 2014. The original plot of this series is not linear and not stationary at levels. Obviously the stochastic properties of the series are also not constant. This suggests that the proposed methods for this study will work well with the data. A perusal of the actual data also shows that from the third quarter of 2002 to the fourth quarter of 2003, inflation rates were very high in South Africa. A sudden dip as low as $4 \%$ followed in the first quarter of 2005 but gradually increased until the highest peak ever which is visible in the third and fourth quarters of 2008. These time-epochs constitute volatility in inflation rates.

\section{Figure 1: Log transformed plot of the South African Inflation rates from Q3 2002 to Q4 2014}

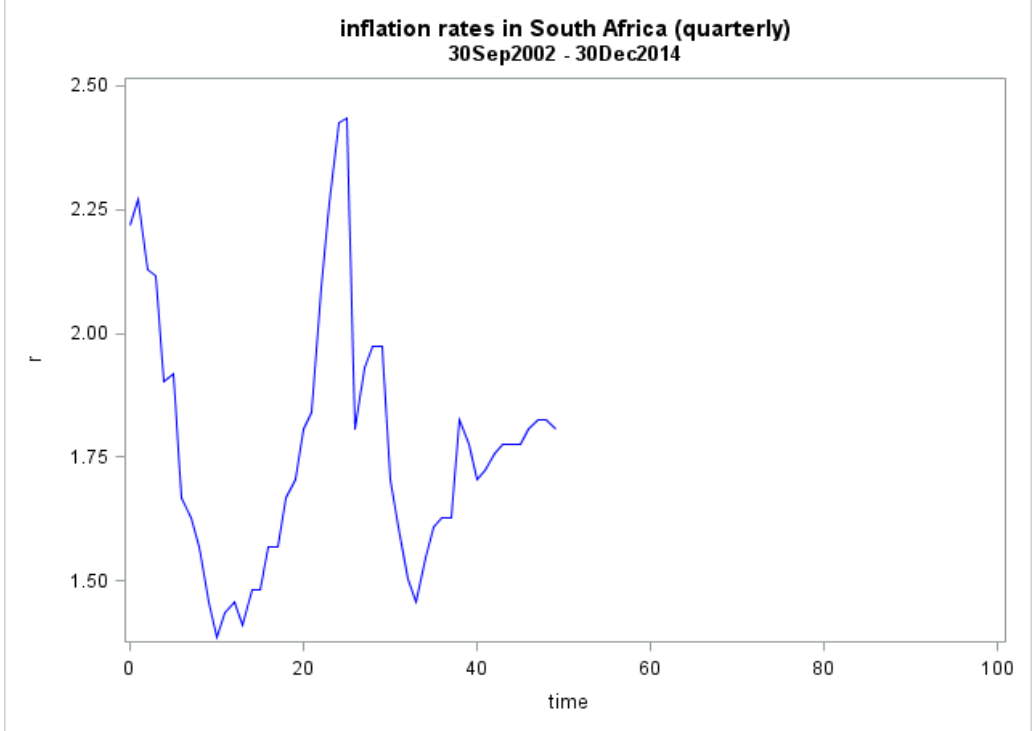

Figure 2 depicts a first differenced inflation series. Visible spikes in this figureare evidence of obvious volatility clustering in the series. This further shows the returns to be moderately stable overtime as results of transformation. The said behaviour of series returns appear to be in line with most financial theories and models which usually assume the prices to returns to be a stationary process. A further analysis is continued with a first differenced stationary series. 
Figure 2: Log transformed-first differenced series of the South African Inflation rates

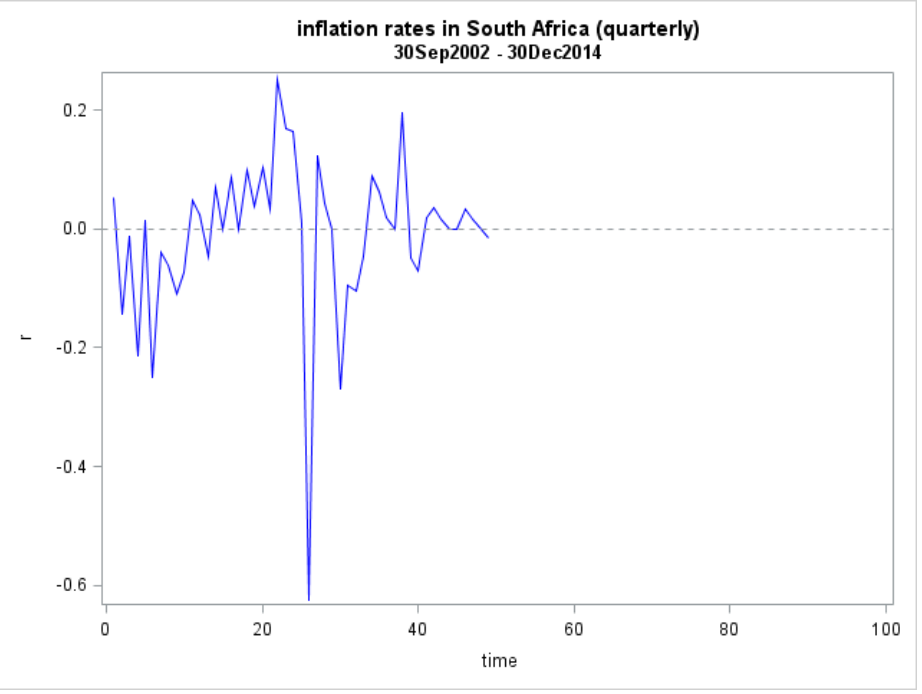

The results in Table 1 show that the AR (1) and the intercept are significant allowing the analysis to be done on the basis of this model as mean equation and GARCH $(1,1)$ as variance equation. Further preliminary analyses provide evidence that the variance changes across time(heteroscedastic) as strongly suggested by the LM statistic in Table 2. The observed probabilities associated with this test are all less than the 0.05 significance level. This is a good indication that a long memory period GARCH model is one approach to modelling time series with heteroscedastic errors than the ARCH model. Further note that the observed Qstatistics in Table 2 indicate that the residuals are non-linear, confirming that the GARCH model would be more appropriate to be used in parsimoniously predicting model volatility error variance than the ARCH model. By observation, the ACF and PACF plots of the residuals provide values which are within bounds of $95 \%$ confidence interval for Gaussian white noise. This strongly suggests that these plots are insignificant at 0.05 significance level thereby confirming that means and variance equations are correctly specified.

Table 1: AR (1) parameter estimates

\begin{tabular}{llllll}
\hline $\begin{array}{l}\text { Parameter Estimates } \\
\text { Variable }\end{array}$ & DF & Estimate & Standard Error & t Value & $\begin{array}{l}\text { Approx } \\
\text { Pr }>|\mathbf{t}|\end{array}$ \\
\hline Intercept & 1 & 6.3680 & 0.7951 & 8.01 & $<.0001$ \\
AR1 & 1 & -0.8386 & 0.0752 & -11.15 & $<.0001$ \\
\hline
\end{tabular}

Table 2: Tests for ARCH Disturbances Based on OLS Residuals

\begin{tabular}{lllll}
\hline Order & $\mathbf{Q}$ & $\mathbf{P r}>\mathbf{Q}$ & $\mathbf{L M}$ & $\mathbf{P r}>\mathbf{L M}$ \\
\hline 1 & 20.4679 & $<.0001$ & 18.5405 & $<.0001$ \\
2 & 21.9400 & $<.0001$ & 22.9840 & $<.0001$ \\
3 & 22.1355 & $<.0001$ & 23.0297 & $<.0001$ \\
4 & 23.1929 & 0.0001 & 23.4135 & 0.0001 \\
5 & 24.4069 & 0.0002 & 23.4429 & 0.0003 \\
6 & 25.3829 & 0.0003 & 23.5906 & 0.0006 \\
7 & 25.6769 & 0.0006 & 23.6034 & 0.0013 \\
8 & 25.6774 & 0.0012 & 23.6050 & 0.0027 \\
9 & 25.7500 & 0.0022 & 23.6238 & 0.0049 \\
10 & 25.8266 & 0.0040 & 23.6271 & 0.0087 \\
11 & 25.8458 & 0.0068 & 23.6528 & 0.0143 \\
12 & 25.8672 & 0.0112 & 23.6631 & 0.0226 \\
\hline
\end{tabular}

The purpose of this study is explore the performance of GARCH-family models and use the selected model in estimating and forecasting inflation volatility in South Africa. To help decide on the GARCH-model best suited 
for the study, the minimum information criteria are used. The selection of the best model was based on the minimum value of the AIC and SBC. The results (not shown here) revealed that the $A R$ (1)_IGARCH $(1,1)$ outperformed all the eight GARCH-type models. Both the AIC and the SBC were in favour of this model. The next selected models were the $G J R-G A R C H(1,1)$ and EGARCH respectively. QGARCH $(1,1)$ was the least favoured model.

Primary Analysis: Since the study sought to explore the ability of GARCH-type models in forecasting inflation volatility, both the symmetric and asymmetric GARCH-type models are considered. Ultimately, an optimal model with least forecast errors will be chosen. Note that the researcher included the mean and the AR effects when fitting the models. This was done to allow the output that contains both the mean and the variance equations. Bollerslev et al. (1992) suggested $\operatorname{GARCH}(1,1)$ and $\operatorname{GARCH}(2,1)$ as adequate for modelling volatilities even over long sample periods. Therefore these models are considered when generating the results. To eliminate biasedness, the models are once more checked for appropriates after including the first and second lags in the GARCH and the results are summarised in Table 3.

Table 3: Selection of most appropriate $\operatorname{GARCH}(m, s)$

\begin{tabular}{lll}
\hline Model & SBC & AIC \\
\hline IGARCH $(1,1)$ & $\mathbf{- 5 0 . 6 2 7 9}$ & $\mathbf{- 5 8 . 1 9 5 5}$ \\
IGARCH $(2,1)$ & -46.7362 & -56.1953 \\
GJR-GARCH $(1,1)$ & $\mathbf{- 7 5 . 3 1 6 1}$ & $\mathbf{- 7 7 . 2 0 7 9}$ \\
GJR-GARCH $(2,1)$ & -34.0793 & -49.2139 \\
EGARCH $(1,1)$ & -47.1374 & -58.4883 \\
EGARCH $(2,1)$ & $\mathbf{- 5 2 . 3 3 4 7}$ & $\mathbf{- 6 5 . 5 7 7 4}$ \\
\hline
\end{tabular}

AR (1)_IGARCH $(1,1), \quad A R(1) \_G J R-G A R C H(1,1)$ and $A R(1) \_E G A R C H(2,1)$ are considered most appropriate among the $\operatorname{GARCH}(m, s)$ models according to the SBC and AIC. Therefore, these models are appropriately fitted as follows;

$$
\sigma_{t}^{2}=\theta_{0}+\phi_{1} \sigma_{t-1}^{2}+\left(1-\phi_{1}\right) a_{t-1}^{2}, \quad \text { for } 1>\phi_{1}>0 .
$$

Please take note that the unconditional variance of $r_{t}=a_{t}+\mu_{t}$, where $a_{t}=\sigma_{t} \epsilon_{t}$ is not defined, and this makes the $A R$ (1)_IGARCH $(1,1)$ model suspect for an excess returns series. The results of the estimated parameters are presented in Table 4.

Table 4:AR (1)_IGARCH $(1,1)$ Estimates

\begin{tabular}{lll}
\hline Variable & Estimate & $\begin{array}{l}\text { Approx } \\
\text { Pr }>|\mathbf{t}|\end{array}$ \\
\hline Mean equation & & \\
Intercept & -0.0014 & 0.9025 \\
AR1 & 0.2936 & 0.0532 \\
Variance equation & & \\
ARCH0 & $1.0537 \mathrm{E}-8$ & $<.0001$ \\
ARCH1 & 0.3540 & $<.0001$ \\
GARCH1 & 0.6460 & $<.0001$ \\
Diagnostics & & \\
Normality & $7.9133(0.0191)$ \\
MSE & 201.445 \\
Total R-Square & Undefined \\
Unconditional & Undefined \\
variance & & \\
\hline
\end{tabular}

The mathematical form of this model is shown as;

$$
\left.\sigma_{t}^{2}=0.0000+0.354 \sigma_{t-1}^{2}+0.646\right) a_{t-1}^{2}, \quad \text { with } 1>\phi_{1}>0
$$




$$
r_{t}=-0.0014+\mu_{t}
$$

The GJR-GARCH model is a simple extension of GARCH with an additional term added to account for possible asymmetries (Knight and Satchell, 2003). AR (1)_GJR - GARCH $(1,1)$ could not be properly fitted. Only the intercept of this model was generated and therefore the $A R(1) G J R-G A R C H(2,1)$ model was generated instead. An appropriate form of this model is given as;

$$
\begin{array}{r}
\sigma_{t}^{2}=\theta_{0}+\phi_{1} \sigma_{t-1}^{2}+\phi_{2} \sigma_{t-2}^{2}+\theta_{1} n_{t-1}^{2}+\theta_{2} n_{t-2}^{2}+\gamma \lambda_{t-1} \varepsilon_{t-1} \\
\lambda_{t-1}=\left\{\begin{array}{ll}
1 & \text { if } \varepsilon_{t-1}<0 \\
0 & \text { if } \varepsilon_{t-1} \geq 0
\end{array}\right\} .
\end{array}
$$

Estimates of parameters of $A R(1) \_G J R-\operatorname{GARCH}(2,1)$ are summarized in Table 5.

\section{Table 5: $A R(1) \_G J R-G A R C H(2,1)$ parameter estimates}

\begin{tabular}{lll}
\hline Variable & Estimate & $\begin{array}{l}\text { Approx } \\
\mathbf{P r}>|\mathbf{t}|\end{array}$ \\
\hline Mean equation & & \\
Intercept & 0.0037 & 0.9401 \\
AR1 & -0.3253 & $\cdot$ \\
Variance equation & & \\
TARCHA & 0.0180 & 0.5394 \\
TARCHA1 & -0.1773 & $\cdot$ \\
TARCHB1 & 0.2448 & 0.5312 \\
GJR-GARCH1 & 0.0108 & $\cdot$ \\
GJR-GARCH2 & 0.005556 & $\cdot$ \\
Diagnostics & & \\
Normality & $170.6632(<0.0001)$ \\
Total R-Square & Undefined \\
Unconditional & 0.0197 \\
variance & & \\
\hline
\end{tabular}

Please note that no leverage effect for $A R(1) \_G R-G A R C H(2,1)$ model could be estimated according to Table 5. Mathematical form of this model is given as;

$$
\sigma_{t}^{2}=0.0195-0.2371 \sigma_{t-1}^{2}+0.2943 \sigma_{t-2}^{2}+0.0943 n_{t-1}^{2}+0.00000 n_{t-2}^{2} .
$$

Table 6 gives results for the AR (1)_EGARCH $(2,1)$ model.

Table 6:AR (1)_EGARCH $(2,1)$ parameter estimates

\begin{tabular}{lll}
\hline Variable & Estimate & $\begin{array}{l}\text { Approx } \\
\text { Pr }>|\mathbf{t}|\end{array}$ \\
\hline Mean equation & & \\
Intercept & -0.0035 & 0.7714 \\
AR1 & -0.0921 & 0.0523 \\
Variance equation & & \\
EARCH0 & -11.6754 & $<.0001$ \\
EARCH1 & 0.5496 & 0.0475 \\
EGARCH1 & -1.0949 & $<.0001$ \\
EGARCH2 & -0.0990 & 0.6399 \\
THETA & -0.1513 & 0.4910 \\
Diagnostics & & \\
Normality & $0.5692(0.7523)$ \\
Total R-Square & Undefined \\
MSE & 180.988 & \\
Unconditional & Undefined & \\
variance & & \\
\hline
\end{tabular}


Mathematically, AR (1)_EGARCH $(2,1)$ model is formulated as;

$$
\log \sigma_{t}^{2}=-11.6754+0.5496 \frac{\left|a_{t-1}\right|+(-0.1513) a_{t-1}}{\sigma_{t-1}}-1.0949 \sigma_{t-1}^{2}-0.0990 \sigma_{t-1}^{2} .
$$

Presented next are the results of the BDS test of serial correlation for the first ten dimensions. A distance of two was used to generate the residuals. The BDS test is used to identify GARCH type that best models the conditional variance of inflation series for the chosen period. The BDS test for serial independence is a nonparametric test used also to detect non-linearity in the standardised residuals of the GARCH-type models selected in this study. This test is based on the null hypothesis that the data are pure white noise, that is, completely random (Broock et al., 1996). Panagiotidis (2002) suggested that once linear or non-linear structure in the data has been removed; the rest should be due to an unknown non-linear data generating process. The expectation is to have the residuals which follow a white noise process to conclude that the model is a true data generating process. The null hypothesis should not be rejected in order to render the model adequate for capturing all the relevant features of the data.

Table 7: BDS Test results for Independence

\begin{tabular}{lllllll}
$\begin{array}{l}\text { Embedding } \\
\text { Dimension }\end{array}$ & AR (1)_IGARCH $(1,1)$ & \multicolumn{2}{l}{ AR (1)_GJR-GARCH $(2,1)$} & \multicolumn{2}{l}{ AR (1)_EGARCH $(2,1)$} \\
& BDS & Pr $>\mid$ BDS $\mid$ & BDS & Pr > |BDS $\mid$ & BDS & Pr > |BDS $\mid$ \\
\hline 2 & -1.2026 & 0.2291 & -0.4556 & 0.6487 & 1.4176 & 0.1563 \\
3 & -1.0990 & 0.2718 & -0.5670 & 0.5707 & 1.1812 & 0.2375 \\
4 & -1.0623 & 0.2881 & -0.2788 & 0.7804 & 1.9385 & 0.0526 \\
5 & -1.4403 & 0.1498 & -0.5974 & 0.5502 & 2.1562 & 0.0311 \\
6 & -0.5703 & 0.5685 & -0.0611 & 0.9513 & 2.2649 & 0.0235 \\
7 & -1.1585 & 0.2467 & -1.3315 & 0.1830 & 1.5941 & 0.1109 \\
8 & -0.7476 & 0.4547 & -1.0572 & 0.2904 & 1.6405 & 0.1009 \\
9 & -0.4672 & 0.6404 & -0.9181 & 0.3586 & 1.5224 & 0.1279 \\
10 & -0.6269 & 0.5307 & -1.0109 & 0.3121 & 1.1228 & 0.2615 \\
\hline
\end{tabular}

Results in Table 7 reveal that the residuals of the three models are not statistically significant at $0.01,0.05,0.1$ levels of significance except for some in $A R(1) \_E G A R C H(2,1)$ model at dimensions 4,5 and 6 . Therefore, the three models satisfy the assumption of randomness of the residuals according to Broock et al. (1996).

Evaluating forecasting ability: This section evaluates the inflation forecast ability by the three models. Generated by SAS are the plots of conditional variances for the selected period and the next 12 months. The associated forecast error measure such as the MAPE, MAE and the MSE are given to help decide on an optimal model. The model with a least forecast error measure is considered robust. The conditional standard deviations are summarised as Figures 3 to 5 and associated measures in Table 8.

Figure 3: $A R(1) \_I G A R C H(1,1)$ Conditional standard deviations

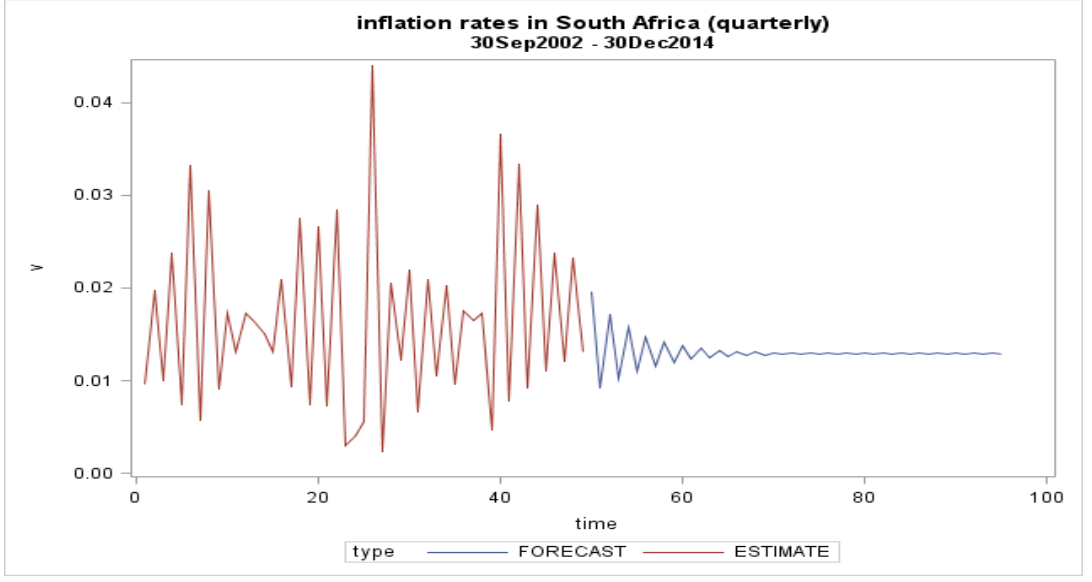


Figure 4 : $A R(1) \_E G A R C H(2,1)$ Conditional standard deviations

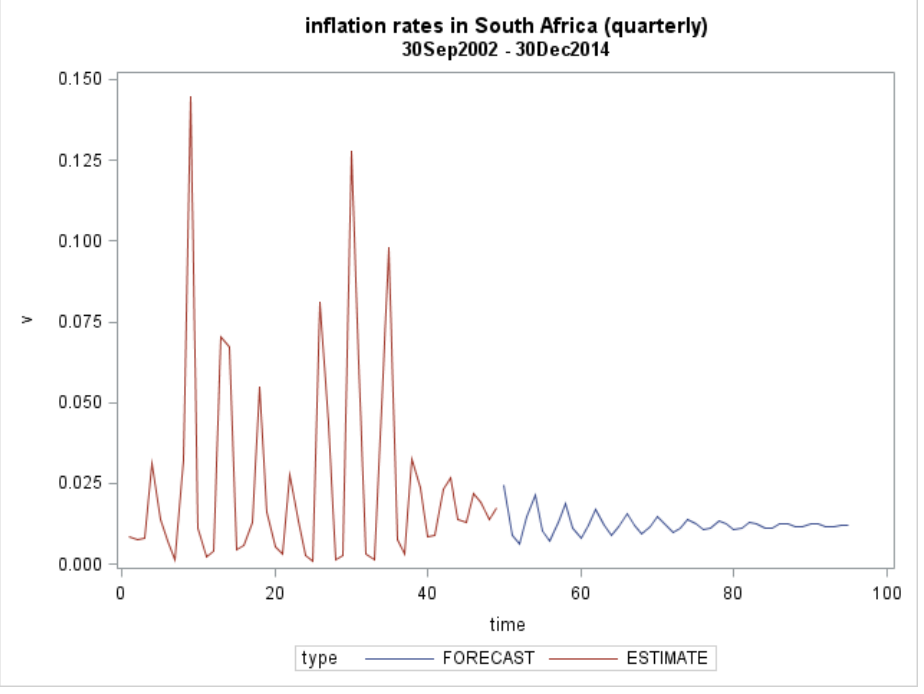

Figure 5: $A R(1) \_G J R-G A R C H(2,1)$ Conditional standard deviations

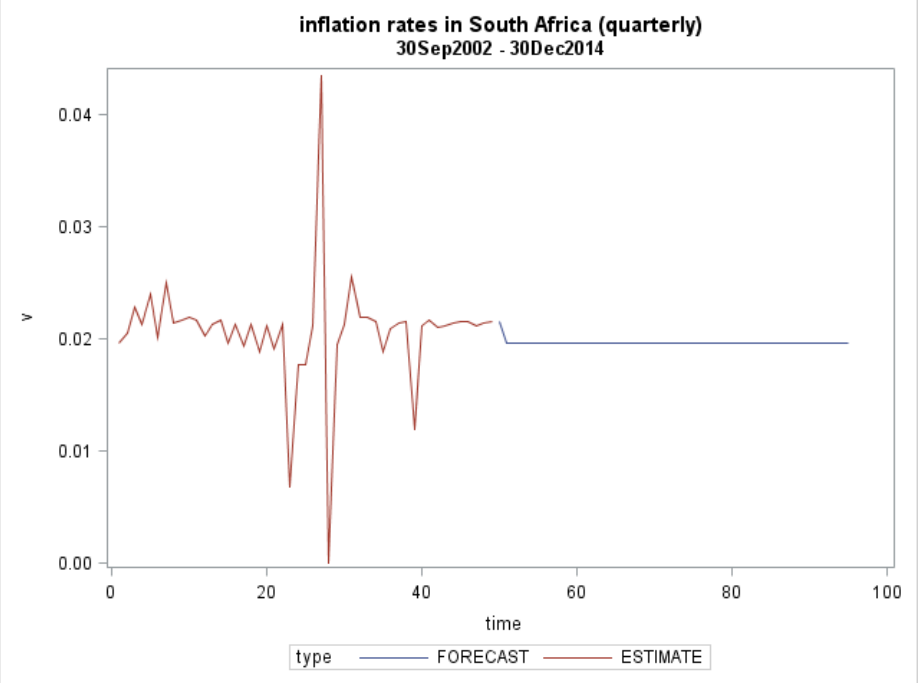

Figure 3 to 5 reveal the forecasts of inflation volatility in the next year, 2015. It is apparent that the error terms in inflation series have not been constant over the years and this is explained well by the spikes visible in the figures. This is also an indication that the South African inflation series is volatile and this is captured well by the three models. However, the forecasts show that in future, inflation may be stable and this is indicative of almost smoothed line depicted in the figures. Both the $A R(1) \_I G A R C H(1,1)$ and $A R(1) \_G J R G A R C H(2,1)$ appear not to be good forecasters of conditional variance.

Table 8: Forecast error measures

\begin{tabular}{llll}
\hline Measure & Model & & \\
& IGARCH (1, 1) & EGARCH $(\mathbf{2 , 1 )}$ & GJR-GARCH (2, 1) \\
\hline MAE & 0.0953 & 0.0814 & 0.0823 \\
MAPE & 146.818 & 102.2620 & 111.8973 \\
MSE & 0.0201 & 0.0177 & 0.0190 \\
$\begin{array}{l}\text { Model } \\
\text { ranking }\end{array}$ & 3 & 1 & 2 \\
\hline
\end{tabular}


The error measures choose the asymmetric AR $(1)$ EGARCH $(2,1)$ model as one which best models conditional variance. More evidence can be gathered on the associated Figure 4 which shows the forecasted volatility by this model. The AR (1)_IGARCH $(1,1)$ model was least ranked by the forecast measures. These findings confirm those reported by many studies on this subject. Therefore, EGARCH is an optimal model among other GARCH-type models.

Figure 6: Forecasting ability of the $A R(1)_{-} E G A R C H(2,1)$ model

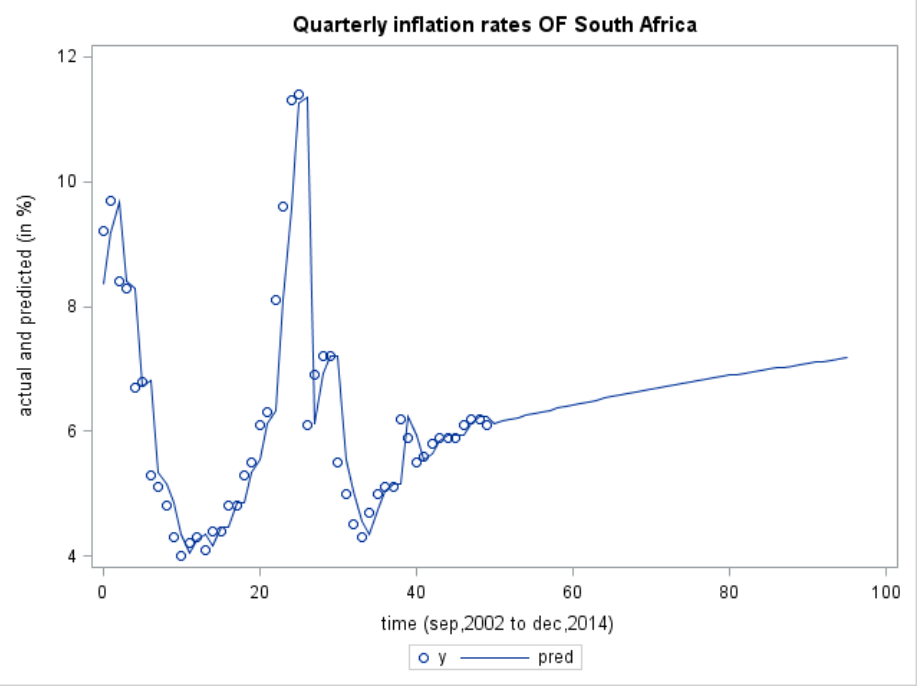

Figure 6 confirms that the $A R(1) E G A R C H(2,1)$ model has the ability to capture the volatility present in the data set. The predicted values are shown to be moving along with the original series. As shown in the figure, inflation volatility forecasts by the model for the period January 2015 to December 2015 will decrease but at a very low pace. The results in Figure 6 confirm the forecasts by the three models in Figure 3 to 5.

\section{Results and Discussion}

Parameter estimates of the $A R(1)$ IGARCH $(1,1)$ for the variance equation are robust as seen in Table 4 . The sum of the ARCH and GARCH coefficients is very close to unity, and this implies that volatility shocks are pretty persistent. This is an indication of a covariance stationary model with a high degree of persistence and long memory in the conditional variance. Due to this high rate, the response function to shock is likely to die slowly. As a result, new shocks may have influence in returns for a longer period. This is another one of the conditions of the GARCH-type model application. Evidence about parameter estimates robustness can be gathered on $p$-values which are less thanthe $0.05,0.01$ and 0.1 levels of significance. The significant parameter estimates of the $A R(1,1)_{-} I G A R C H$ model further proofs that coefficients of the conditional variance equation have a strong support for the presence of ARCH and GARCH effects. The AR term in the mean equation of this model is significant at 0.1 level of significance. The results in Table 4 also suggest that the intercept (also known as the mean) of the return series is not significantly different from zero. This condition is consistent with the random walk hypothesis. The leverage effect in the mean equation exceeds zero and is significant at 0.1 level of significant. This is in support of the assumption that negative and positive shocks have different impact on the volatility quarterly returns.

The variance equation parameters of the $A R(1) \_E G A R C H(2,1)$ model in Table 6are significant except for one associated with EGARCH2. The leverage effect is calculated as -0.1513 and is negative and insignificant as expected.The asymmetric (leverage) effect captured by the negative parameter estimate is less than zero suggesting absence of leverage effect. Parameter estimates of the mean equation for the $A R(1) \_E G A R C H(2,1)$ model are insignificant.Parameter estimates of the $A R(1)$ GJR - GARCH $(2,1)$ model in Table 5 both for the mean and variance equations seem to be unstable and insignificant. Due to inconclusive results exhibited by this model, not much can be deliberated. The mean values in the mean equations of the three models are less than zero and insignificant, implying that these models detect low volatility in inflation 
series. This also explains why the spikes in Figures 3 to 6 are gradually levelling off. This may be good news to the country as many sectors affected by this series may be stabilised. Volatility has disadvantages such as the potential to unsettle investors and undermining the role of exports in growth strategy of economies. The findings of this study provide a good motivation for the implementation of fixed inflation rates in future and consequently consumer prices may be lowered and purchasing power of money may be improved. This will enable more people to start saving for the future since they will have more money left in their pockets.

The diagnostic tests of the three models provide significant observed probabilities of the normality tests for the $A R(1) \_G J R-G A R C H(2,1)$ and $A R(1) \_I G A R C H(1,1)$ models. The assumption of normality is violated for these models but catered for by the AR $(1)$ EGARCH $(2,1)$ model. The normality test in the first two models indicates that the conditional normal distribution may not fully explain the leptokurtosis in inflation rates but is fully supported by the AR (1)_EGARCH $(2,1)$ model (Bollerslev, 1987). The residuals of the three models are also independent according to the BDS test summarised in Table 8 for all embedding dimensions implying that the models are correctly specified. The residuals of the three models also follow a white noise process, implying these models are a true data generating process. Further diagnostics tests provide the results for the forecast error measures which are all in favour of the $A R(1) E G A R C H(2,1)$ model. The diagnostic tests and the absence of leverage or asymmetric effects confirm that the $A R(1) E G G A R C H(2,1)$ model is best suited to measure the volatility of the inflation series. The findings are in accordance with those reported by different studies. EGARCH model outperforms other GARCH models as it models and forecasts volatility better than other GARCH-type models.

\section{Conclusion}

This paper explored the robustness of GARCH-type models in estimating the volatility of inflation rates in South Africa. Volatility clustering, leptokurtosis and leverage effects were examined for the series for the period 2002 September to December 2014. Preliminary analyses proved that the data has heteroscedastic and non-linear errors. Stationarity of the series was achieved after first differencing the log transformed data. About nine GARCH-type models were compared and only three were selected according to the AIC and SBC. The maximum likelihood estimation method was used to estimate parameters of the selected models and it was discovered that $A R(1) \_G J R-G A R C H(2,1)$ was the least robust model in terms of the parameter estimates and other diagnostic tests. Though the sum of ARCH and GARCH effects in the $A R$ (1)_IGARCH $(1,1)$ model were in accordance with theory and significant compared to the $A R$ (1)_EGARCH $(2,1)$ model, the former was ranked second by the minimum information criteria. The intercepts of the three models were very small (less than zero) and insignificant implying that volatility effects captured by these models in inflation rates are very low.The leverage effect in the $A R$ (1)_EGARCH $(2,1)$ model suggested absence of leverage effect in the series. Overall, the $A R(1)$ EGARCH $(2,1)$ model was found to be more appropriate for modelling the series with heteroscedastic errors. This model proved that inflation rates in South Africa exhibits the stylised characteristics such as volatility clustering, leptokurtosis and asymmetry effects.

The parameter estimates of the AR (1)_IGARCH $(1,1)$ model suggested a high degree persistent in the conditional volatility of the series. This evidence of high volatility persistence and long memory in this model is suggestive of relevant IGARCH models probably with ARMA effects. Other asymmetric GARCH models may be used for further studies and the results may be compared with those obtained in this study. This may aid in identifying the most optimal model among the family of asymmetric heteroscedastic models that are more appropriate for inflation rates. The forecasts obtained for inflation rates obtained through the AR (1)_EGARCH $(2,1)$ model could be of vast help to decision makers when formulating strategies concerning this sector. The information obtained would go a long way in arriving at decisions on issues such as augmentation of consumer prices in future. Decision makers could also be able to, from time to time well in advance, benchmark with other countries and prepare for the future. 


\section{References}

Alnaa, S. E. \& Ahiakpor, F. (2011). ARIMA approach to predicting inflation in Ghana. Journal of Economic and International Finance, 3(5), 328-336.

Amos, C. (2010). Time Series Modelling with Applications to South African Inflation Data. (Unpublished master's thesis) submitted to University of Kwazulu Natal, Kwa.zulu Natal.

Awogbemi, C. A. \& Oluwaseyi, A. (2011). Modeling volatility in financial time series: Evidence from Nigerian inflation rates. Ozean Journal of Applied Science, 4(3), 337- 350.

Bollerslev, T. (1986).Generalized autoregressive conditional heteroskedasticity. Journal of Econometrics, 31, 307-327.

Bollerslev, T. (1987).A conditional heteroscedastic time series model for speculative prices and rates of return. Review of Economics and Statistics, 69, 542-547.

Bollerslev, T., Chou, R. Y. \& Kroner, K. F. (1992).ARCH Modelling in Finance: A Selective Review of the Theory and Empirical Evidence. Journal of Econometrics, 52, 5-59.

Brock, W. A., Hsieh, D. \& LeBaron, B. (1991). Nonlinear Dynamics, Chaos, and Instability: Statistical Theory and Economic Evidence. MIT Press, Cambridge, Massachusetts.

Broock, W. A., Scheinkman, J. A., Dechert, W. D. \& LeBaron, B. (1996). A Test for Independence Based on the Correlation Dimension. Econometric Reviews, 15(3), 197-235.

Chong, C. W., Loo, C. S. \& Muhammad, A. M. (2002).Modelling the Volatility of Currency Exchange Rates Using GARCH Model. Putra, Malaysia Press.

David, F. H. (2001). Modeling UK inflation, 1875-1991. Journal of Applied Econometrics, 16(3), 255-275.

Ding, Z., Granger, C. W. J. \& Engle, R. (1993). A Long memory property of stock market returns and a new model. Journal of Empirical Finance, 1, 83-106.

Dong, Y. (2012). ARMA and GARCH-type modelling electricity prices, Masters thesis submitted to Chalmers University of Technology, Sweden.

Engle, R. F. \& Ng, V. K. (1993).Measuring and testing the impact of news on volatility. Journal of Finance, 48, $1749-1778$.

Engle, R.F. (1982). Autoregressive conditional heteroscedasticity with estimates of the variance of United Kingdom inflation. Econometrica, 50, 987-1007.

Faraway, J. \& Chatfield, C. (1998). Time series forecasting with neural networks: A comparative study using the airline data. Applied Statistics, 47, 231- 250.

Frimpong, J. M. \& Oteng-Abayie, E. F. (2006). Modelling and forecasting volatility of returns on the Ghana Stock Exchange Using GARCH Models. American Journal of Applied Sciences, 3(10), 2042-2048.

Glosten, L., Jaganathan, R. \& Runkle, D. (1993).Relationship between the expected value and volatility of the nominal excess returns on stocks. Journal of Finance, 48, 1779-1802.

Hsieh, D. (1991). Chaos and nonlinear dynamics: Application to financial markets. Journal of Finance, 46, 1839-1877.

Igogo, T. (2010). Real exchange rate volatility and international trade flows in Tanzania, A Dissertation submitted at the University of Dar es Salaam in Partial Fulfilment of the requirement for the degree of M.A (Economics).

Jehovanes, A. (2007).Monetary and inflation dynamics: A lag between change in money supply and the corresponding inflation responding in Tanzania. Monetary and financial affairs. Department Bank of Tanzania, Dar Es Salaam

Knight, J. L. \& Satchell, S. (2003). Forecasting volatility in the financial markets, $2^{\text {nd }}$ ed. Singapore: Wiley and Sons Ltd.

McLeod, A. I. \& Li, W. K. (1983). Diagnostic checking of ARMA Time series models using squared-residual autocorrelations. Journal of Time Series Analysis, 4, 269-273.

Mokoma, T. J. \& Moroke, N. D. (2015). Is the South African exchange rate volatile? Application of the ARCH framework. Risk Governance and Control Journal, 5(1), 110-122.

Nelson, D. B. (1991).Conditional heteroskedasticity in asset returns: A new approach. Econometrica, 59, 347370.

Ngailo, E. (2011). Modelling and forecasting using time series GARCH models: An application of Tanzania inflation rate data. Masters dissertation submitted to the University of Dar es Salaam.

Nortey, E. N. N., Mbeah-Baiden, B., Dasah, J. B. \& Mettle, F. O. (2014).Modelling Rates of Inflation in Ghana: An Application of Arch Models. Current Research Journal of Economic Theory, 6(2), 16-21. 
Osarumwense, O. \& Waziri, E. I. (2013). Modeling monthly inflation rate volatility, using Generalized Autoregressive Conditionally Heteroscedastic (GARCH) models: Evidence from Nigeria. Australian Journal of Basic and Applied Sciences, 7(7), 991-998.

Panagiotidis, T. (2002).Testing the Assumption of Linearity. Economics Bulletin, 3(29), 1-9.

Perniaag, F. E., Nor, M. \& Ekonomi, P. P. (2004), Modelling and Forecasting on the Malaysian inflation Rates: An Application of GARCH Models, Bangi, UKM press.

Scheinkman, J. A. \& LeBaron, B. (1989). Nonlinear dynamics and GNP Data," in Economic complexity: chaos, sunspots, bubbles, and nonlinearity, ed. by W. A. Barnett, J. Geweke, and K. Shell. Cambridge University Press.

Su, C. (2010). Application of EGARCH Model to Estimate Financial Volatility of Daily Returns: The empirical case of China. (Unpublished master's thesis submitted to the University of Gothenburg, Sweden.

Suleman, N. \& Sarpong, S. (2012). Empirical approach to modeling and forecasting inflation in Ghana. Current Research Journal of Economic Theory, 4 (3), 83-87.

Tsay, R. S. (2005). Analysis of financial time series. $2^{\text {nd }}$ ed. New York, John Wiley \& Sons.

Tsay, R. S. (2010). Analysis of financial time series, $3^{\text {rd }}$ ed., New York, John Wiley \& Sons.

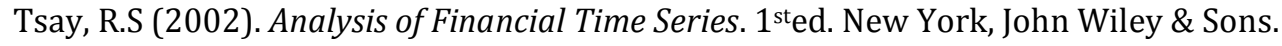

Webster, D (2000). Webster's new universal unabridged dictionary. Barnes and Noble Books, New York.

Wei, W. W. S. (2006). Time series analysis: univariate and multivariate analysis, $2^{\text {nd }}$ ed. Edison, Wesley Pearson. Weiss, A. A. (1984). ARMA models with ARCH errors. Journal of Time Series Analysis, 5, 129 - 143.

Zakoian, J. M. (1994). Threshold heteroscedastic models. Journal of Economic Dynamics and Control, 18, 931955. 